\title{
The Utilization of Hyperspectral Imaging for Impurities Detection in Secondary Plastics
}

\author{
S. Serranti ${ }^{*}$ A. Gargiulo and G. Bonifazi \\ Department of Chemical Engineering Materials \& Environment, Sapienza University of Rome, Via Eudossiana 18, \\ 00184, Rome, Italy
}

\begin{abstract}
The systematic identification of impurities inside secondary plastics flow streams can be considered as one of the key issues to certify and to classify waste plastics fed to recycling plants and to perform a full control of the resulting processed fractions, that have to comply with market demands on grade and purity of the recovered products when compared with virgin streams. HyperSpectral Imaging (HSI) can represent an optimal, reliable and low costs answer to reach the previous mentioned goals. HSI is based on the utilization of an integrated hardware and software architecture able to digitally capture and handle spectra, as an image sequence, as they results along a pre-defined alignment on a surface sample properly energized. According to the different wavelengths of the source and the different spectral sensitivity of the device, different physical-chemical superficial characteristics of the sample can be investigated and analyzed. Such an approach, if fully investigated and implemented, could allow to perform a big-step-forward inside quality-inspection-control-strategies/logics (QICSL) applied to the entire waste sector. The identification of contaminants in secondary plastics, adopting HSI, thus can represent a first attempt to introduce new QICSL in an innovative polyolefins separation process from end-of-life product. Such an approach well fit the goals that are at the base of a the application of a non-linear Cyclic Innovation Model (CIM) addressed to connect technical capabilities (contaminants identification) with societal market needs (quality of the recycled products).
\end{abstract}

Keywords: Recycling, secondary plastics, polyolefins, hyperspectral imaging, sorting.

\section{INTRODUCTION}

Used plastic packaging and other plastic items can be valuable resources in the manufacture of new products and in the generation of energy. It is important that as a society we aim to make the best affordable use of these valuable plastic resources. This is good for the environment, for the economy and for the international community. Analyses by the European Community (EU) indicate that besides their ecologic importance, raw materials and energy are also the most important competitiveness factors for EU industries. Therefore the need to increase recycling, improving at the same time the quality and homogeneity of recycled materials to minimize environmental pollution and usage of resources is thus a topical subject for the EC. There is a strong drive to recycle polymers from end-of-life products and avoid their ending up in land fills and waste incinerators because plastics recycling reduces $\mathrm{CO}_{2}$ emission and saves resources. The worldwide production of plastics was 230 million ton in 2005 [1]. In Europe, 53.5 million ton were produced in total. Out of 22 million ton of post-consumer plastic waste in Europe in 2005, 53\% was disposed, 29\% was used for energy recovery and $18 \%$ was recycled [1]. According to the last EU Directive 2004/12/EC on packaging and packaging waste, the recycling level of plastics should dramatically increase in the next years. New, more cost-effective separation technology can thus provide an important

*Address correspondence to this author at the Sapienza - Università di Roma, DICMA, Via Eudossiana n. 18, 00184 Rome, Italy; Tel: +39 06 44585 360; Fax: +39 0644585 618; E-mail: silvia.serranti@uniroma1.it incentive to increase recycling rates. The recycling of polymers that are present in relatively pure streams such as post-industrial waste and separately collected containers of food and beverage is generally well-developed in Europe. The situation is very different for the very large and complex stream of post-consumer waste, including wastes such as Waste from Electrical and Electronic Equipment (WEEE), household waste, Building \& Construction Waste (B\&CW) and Automotive Shredder Residue (ASR). Effective recycling of these wastes is possible, as has been demonstrated at some places in Europe, by large investments in logistics and dismantling (cars, electronic equipment) or hand-sorting (household waste). Such strategies are very expensive, however, and they are therefore not widely applied.

There are about 50 different groups of plastics, with hundreds of different varieties. All types of plastic are recyclable. To make sorting and thus recycling easier, the American Society of Plastics Industry developed a standard marking code to help consumers to identify and sort the main types of plastic. An example of these types and their most common uses are reported in Table $\mathbf{1}$.

Some companies have started up initiatives to process complex wastes by shredding and sink-floating to produce polyolefin concentrates of varying quality. Analysis of such concentrates generally shows a mixture of polyolefin's, rubbers, foams, fibers and wood, next to varying amounts of materials heavier than water. Currently available separation techniques, based on the difference in flotation properties in water, can be used to separate lighter types of plastic such as 
Table 1. Example of Most Common Type of Plastics and Use

\begin{tabular}{|l|l|l|}
\hline & PET & Polyethylene terephthalate - Fizzy drink bottles and oven-ready meal trays. \\
\hline
\end{tabular}

polypropylene (PP), high density polyethylene (HDPE) and low density polyethylene (LDPE) from the heavier types such as polyethylene terephthalate (PET) and polyvinyl chloride (PVC). Even so, PP, HDPE and LDPE together are both difficult to separate and chemically incompatible, making low-quality objects such as traffic bollards, the most common recycling application. In order to produce highpurity granulates from these concentrates, of a quality comparable to materials produced from post-industrial waste, the mixture must be sorted very accurately, and in order to be economically and ecologically sound, most of the polyolefin's should end up in a useful product. Such accurate and efficient separations exist, but they involve multiple separations and are therefore expensive. A known method is to separate the mixtures into five fractions using separation media with densities of $880 \mathrm{~kg} / \mathrm{m}^{3}, 920-930 \mathrm{~kg} / \mathrm{m}^{3}, 940$ $\mathrm{kg} / \mathrm{m}^{3}$ and $970 \mathrm{~kg} / \mathrm{m}^{3}$. Such a procedure will create highpurity PP and HDPE products, whereas foams, most of the wood and rubbers, LDPE, filled PP and residual heavy materials will end up in relatively small residue fractions. Certain expensive liquids have been specially designed to separate at one of the target densities for polyolefin recycling. Other technologies, such as electrostatic separation and thermal adhesion, have been able to create only a single relatively pure product. Electrostatic separation is based on the utilization of frictional charge characteristics which are obtained when different types of plastics are rubbed together so that attractive and repulsive forces take place between the plastics and charged electrodes. Such an approach for its principle (low attractive or repulsive surface forces) is not particularly efficient and it is strongly influenced by the status of the surfaces. Thermal adhesion is a suitable method to remove low concentration of the adhesive polymer, rather than a bulk sorting method for complex mixtures [2]. Preliminary studies demonstrated that the system, even if efficient, need to be fed with simple waste streams, that is streams optimally constituted only by two plastic materials so that an on-off separation principle (plastic particles sliding or melting-sticking) can be applied according to their different melting points. A preliminary good processing is thus required so that to obtain an optimal, almost bi-component, feed.

In this paper a new technology, based on HyperSpectral Imaging (HSI), is described and introduced. This technology can be profitable used in the recycling sector both as smart detection engine for sorting and/or as flow stream quality control. The proposed sensing architecture is fast, accurate, affordable and it can strongly contribute to bring down the economic threshold above which recycling is cost efficient. As outlined in an other paper of this special issue [3], although HSI can be considered very promising, such an innovative approach is meaningful and reasonable to be developed only together with new separation logics and technologies and/or innovative "on-line" control devices able to play on the different waste materials characterising secondary plastics flow streams to recycle. The European FP7 Project W2Plastics - Magnetic Sorting and Ultrasound Sensor Technologies for Production of High Purity Secondary Polyolefins from Waste, represents a challenge to develop and manage innovations avoiding disciplinary segmentation and taking the technological, economic, social and cultural aspects of innovation into account at all stages along the innovation path. In this perspective the introduction of HSI based quality-inspection-controlstrategies/logics (QICSL) inside a non-linear Cyclic Innovation Model (CIM) [4-6] can contribute to strength and enhance the interaction between technical capabilities and market forces in terms of possible success or failure of an innovation project as W2Plastics. The implementation of the HSI technology, in a CIM perspective [7], is discussed in this paper with particular reference to impurities detection in secondary plastics and with the specific aim to fully explore the potentialities of the procedure in order to contribute to improve recycling industry performance.

\section{HYPERSPECTRAL IMAGING}

HyperSpectral Imaging (HSI), known also as chemical or spectroscopic imaging, is an emerging technique that combines the imaging properties of a digital camera with the 


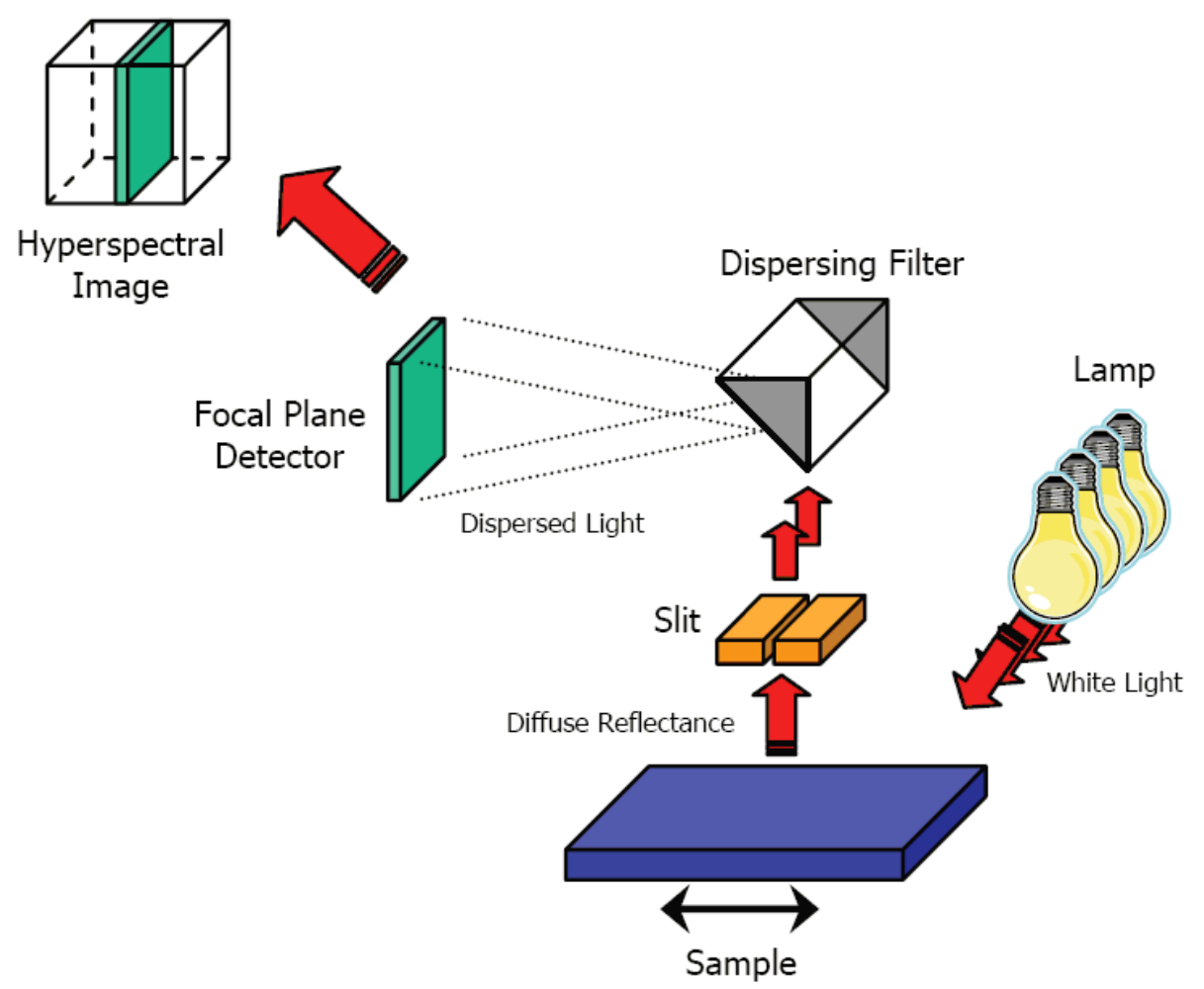

Fig. (1). The hyperspectral camera utilizes a two dimensional detector perpendicular to the surface of the sample to capture the complete spectra from a set of points across the surface of the sample. Hyperspectral images are created from collecting sets of these matrices while repositioning the sample in a direction perpendicular to the sample scan line [8].

spectroscopic properties of a spectrometer able to detect the spectral attributes of each pixel in an image (Fig. 1). Thus, a hyperspectral image, is a three dimensional dataset with two spatial dimensions and one spectral dimension [8].

HSI was originally developed for remote sensing applications [9] but has found application in such diverse fields as astronomy $[10,11]$, agriculture [12-14], pharmaceuticals [15-17] and medicine [18-21]. Some advantages of HSI over conventional true colour imaging (RGB), near infrared imaging (NIR) and multispectral imaging (MSI) are outlined in Table 2. HSI based architectures, in addition to spatial information, can provide spectral information in a wide wavelength range for each pixel of the image [22]. Hyperspectral images are, in fact, made up of hundreds of contiguous wavebands for each spatial position of a target studied.

Table 2. Comparison of RGB Imaging, NIR Spectroscopy (NIRS), Multispectral Imaging (MSI) and Hyperspectral Imaging (HSI) [17]

\begin{tabular}{|l|c|c|c|c|}
\hline Feature & RGB Imaging & NIRS & MSI & HSI \\
\hline \hline Spatial information & $y e s$ & no & yes & $y e s$ \\
\hline Spectral information & no & yes & low & $y \boldsymbol{e s}$ \\
\hline Multi-constituent information & low & $\boldsymbol{y e s}$ & low & $\boldsymbol{y} \boldsymbol{e s}$ \\
\hline Sensitivity to minor components & no & no & low & $\boldsymbol{y} \boldsymbol{e s}$ \\
\hline
\end{tabular}

Each pixel in a hyperspectral image contains the spectrum of that specific position. The resulting spectrum acts like a fingerprint which can be used to characterize the composition of that particular pixel. Hyperspectral images, known as hypercubes [19], are three-dimensional blocks of data, comprising two spatial and one wavelength dimension (Fig. 2). The hypercube allows for the visualization of specific attributes (i.e. physical, chemical, biological, etc.) characteristics of a specific sample, separated into particular areas of the image, since regions of a sample with similar spectral properties have similar "chemical" composition.

It is currently unfeasible to obtain information in all three-dimensions of a hypercube simultaneously; one is limited to obtaining two dimensions at a time, then creating a three-dimensional image by stacking the two dimensional "slices" in sequence. There are two conventional ways to construct a hypercube, the first one known as the "staring imager" configuration and the second known as "pushbroom" acquisition.

The "staring imager" configuration involves keeping the image field of view fixed, and obtaining images one wavelength after another. Hypercubes obtained using this configuration thus consist of a three-dimensional stack of images (one image for each wavelength examined), stored in what is known as the Band Sequential (BSQ) format. Wavelength in the "staring imager" configuration is typically moderated using a tunable filter, Acousto-optic Tunable Filters (AOTFs) and Liquid Crystal Tunable Filters 


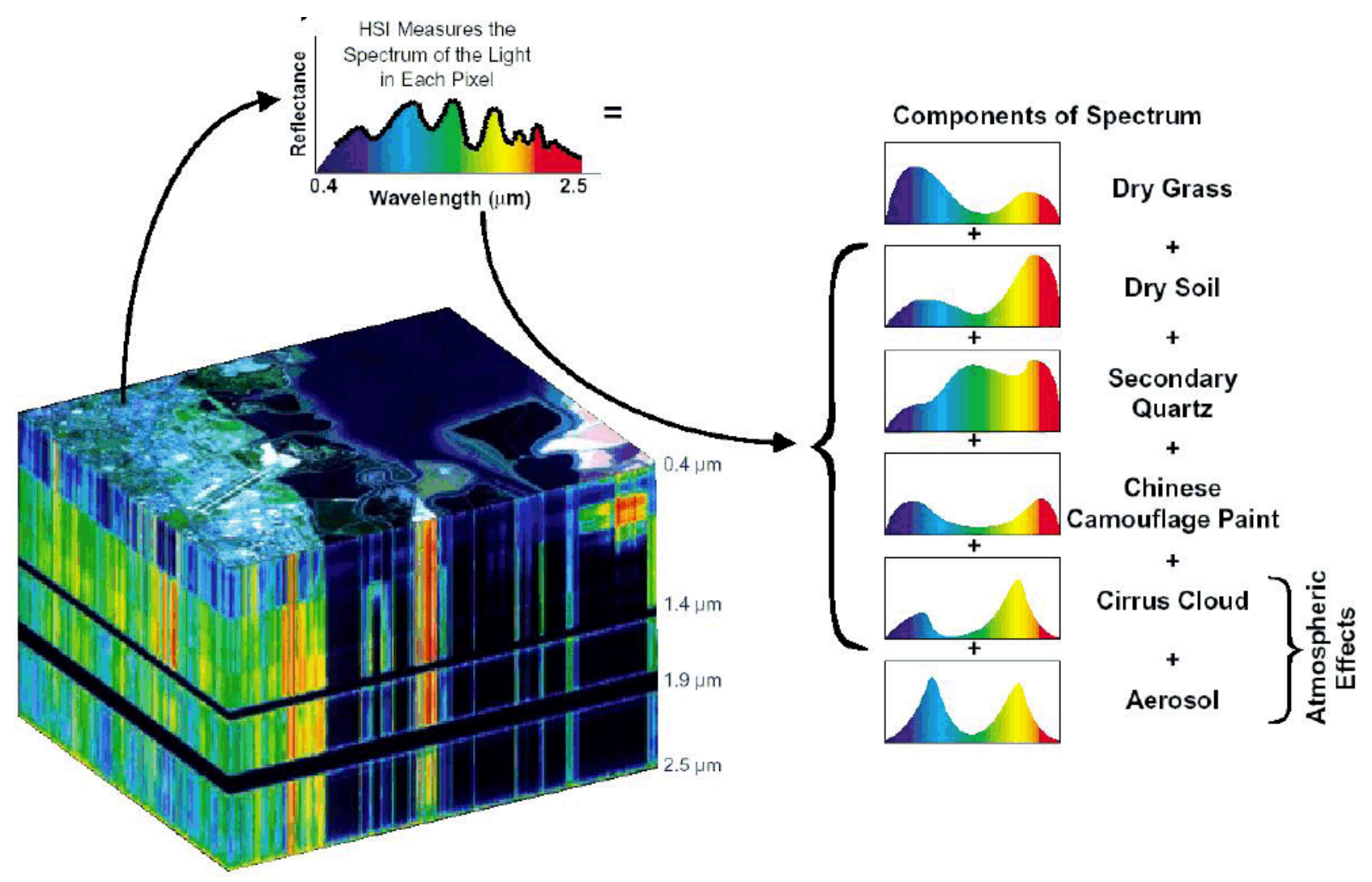

Fig. (2). Example set of scene basis functions based on hyperspectral measurement results [23]. The bottom left figure is a hypercube of data with spatial location given by the $\mathrm{x}$ and $\mathrm{y}$ axes and spectral information contained along the $\mathrm{z}$-axis. The spectral distribution of one pixel is shown in the upper left hand corner and the components that make up that spectrum (along with their physical origin) are shown on the right hand side.

(LCTFs) are the two most predominantly employed. AOTFs have been used in the construction of commercially available NIR-CI systems [24]. The main advantages of AOTFs are good transmission efficiency, fast scan times and large spectral range. On the other hand, LCTFs show greater promise for filtering of Raman images, due to superior spectral band pass and image quality [25]. "Staring imager" instruments incorporating tunable filters have found a number of applications in pharmaceutical quality control [17, 26], their lack of moving parts represents an advantage in many situations.

The "pushbroom" [27] configuration is based on the acquisition of simultaneous spectral measurements from a series of adjacent spatial positions; this requires relative movement between the object and the detector. Some devices produce hyperspectral images based on a point step and acquire mode: spectra are obtained at single points on a sample, then the sample is moved and another spectrum taken. Hypercubes obtained using these configurations are stored in what is known as the $\mathbf{B}$ and Interleaved by Pixel (BIP) format. Advances in detector technology have reduced the time required to acquire hypercubes. Line mapping instruments record the spectrum of each pixel in a line of sample which is simultaneously recorded by an array detector; the resultant hypercube is stored in the $\mathbf{B}$ and
Interleaved by Line (BIL) format. This method is particularly well suited to conveyor belt systems, and may therefore be more practicable than the former for industry applications. The hyperspectral detection architecture that has been utilized in the experimental tests reported in the following belongs to this class of instruments.

This versatile technique has many potential applications in particulate solids characterization where the recognition of finite physical domains of different nature and composition is required. Considering a particle as a relatively small or the smallest discrete portion or amount of something, it is of great importance in many cases to perform a full characterization of it, trying to extract and quantify those physical-chemical attributes influencing its characteristics and behavior. All these aspects can be investigated, in many cases, adopting HSI based techniques. Following these approach a particle can be thus characterized by different spectral signatures in different spectral ranges, from the visible (VIS) to the infrared (NIR) ones. In this paper, specific and ad hoc HSI applications, carried out on different particulate solids products adopting a "pushbroom" based architecture, are reported, analyzed and critically compared in order to highlight the analytical characteristics of the proposed approach. 


\section{HSI RESEARCH APPLICATIONS IN SOLID WASTE RECYCLING}

Different investigations, specifically addressed to waste products characterization, constituents and/or contaminants detection, have been carried out, and are currently investigated, by the authors with reference to different solid waste flow streams. In the following three different applications, related to bottom ashes, light fractions resulting from car dismantling (fluff) and waste glass fragments (cullets) are synthetically reported and discussed. Results are shown according to the Hyperspectral Principal Component Imaging Approach (HPCIA) adopted, that is in terms of image of scores of a specific Principal Component (PCi) and/or associated score plots [28].

\subsection{Bottom Ashes}

Issues: Evaluation of organic matter inside ash residues (bottom ashes) resulting from combustion of Municipal Solid Waste (MSW). Bottom ash consists of inert residues, glass, unburned organic matter and metallic objects and 2 to $20 \%$ carbon. Bottom ash residues, after different and specific processing strategies, mainly based on separation (magnetic and eddy current based) and classification (screening and cycloning) actions can be profitably utilized for fill and road base, but they must comply with strict regulations (civiltechnical and environmental requirements). Its characterization is thus an important step in view of sustainable waste management. In particular, the presence of the so-called "organic matter" fraction represents a strong and severe constraint in respect of their re-use as "common" inert material.

State of the art: Actually, any on-line control architecture does not exist to detect the presence of organic matter inside a bottom ashes flow stream. Bottom ash quality is usually determined at laboratory scale on sample collected in the plant. An "off-line" analysis is performed. Bottom ashes are thus a posteriori certified. At processing plant level is practically impossible to implement control logics inside a bottom ashes processing plant specifically designed to remove the residual organic matter, the fines and the nonferrous metals [29].

Results: The HSI based analyses have been carried out on samples of sand fraction $(-2 \mathrm{~mm})$ resulting from a wet processing, applied to the bottom ash resulting from the Amsterdam MSW incinerator and finalized to obtain a product where the residual organic matter and fines satisfied the Dutch building materials decree [30]. Both chemical and hyperspectral imaging analyses have been carried out, in order to find a correlation between chemical composition of sand product, with particular reference to the organic matter content, and spectral signature in the visible-near-infrared (VIS-NIR) wavelength range: $400-1000 \mathrm{~nm}$, in order to also identify and locate the presence of polluting materials. The results showed as a strict correlation can be established between detected spectral signature intensity of processed bottom ashes samples [29] and topological assessment of organic matter particles (Fig. 3).

\subsection{Light Fractions Resulting from Car Dismantling: Fluff}

Issues: Fluff is the name conventionally used from Automotive Shredded Residues (ASR) light fractions. Fluff represents about the $25 \%$ of the weight of a car and is usually constituted by materials characterized by intrinsic low specific gravity (i.e. plastics, rubber, synthetic foams, etc.). When processed to perform their recovery, fine fluff fractions, below $1 \mathrm{~mm}$, results polluted by materials as copper, aluminum, brass, iron, etc. characterized by shapes (i.e. wires, metal straps, slip rings, wipers, etc.) that does not allow separation by classical physical techniques.

State of the art: The possibility to properly separate and clean the lighter fractions could strongly improves the possibility to set up more efficient recycling strategies, reducing waste disposal and environmental pollution and increasing, at the same time, the energy recovery through pure sorted polymer re-use. Furthermore the possibility to utilize finer fluff fractions to produce energy could contribute to increase the recovery of such a kind of products. To reach this goal, the quantity and the quality of the metal contaminants have to be strongly controlled not to prejudice the quality of the final fluff based fuel.

Results: The hyperspectral approach applied to $-1 \mathrm{~mm}$ fluff fractions allow to perform the recognition of the different materials, selecting specific wavelength bands in the VIS-NIR wavelength field in which the differences in reflectance values are best highlighted [29]. The recognition is particularly robust for metals; considering that these particles represent, not only a fuelling material, but also the main pollutants, the possibility to perform their recognition represents a big step forward to set up innovative selection strategies aimed to produce fine fluff particles to be utilized as a source of energy. Furthermore the possibility to locate them inside the waste flow streams (Fig. 4) opens new scenarios to set up innovative sorting equipments, and quality-control logics, to remove such a kind of pollutants not easy to manage with the commonly utilized physical separation devices.

\subsection{Cullets}

Issues: Identification of ceramic glass contaminants in the cullets (glass fragments) strongly affects the quality of glass recycled products. Such polluting elements, being characterized by higher melting point than glass, can produce severe damages in the production equipment and on the final manufactured goods (bottles, vases, jars, etc.).

State of the art: In glass recycling plants, most contaminants (metals, plastics, wood, and paper) are commonly removed adopting different on-line classification and/or sorting strategies as they are characterized by different physical properties from those characterizing glass fragments. Ceramic glass, presenting similar physical properties to those of glass are practically impossible to identify and separate with the commonly utilized separation and/or automated optical based sorting devices, usually employed for colored cullet separation. Studies carried out adopting X-ray based sorting techniques [31] were 


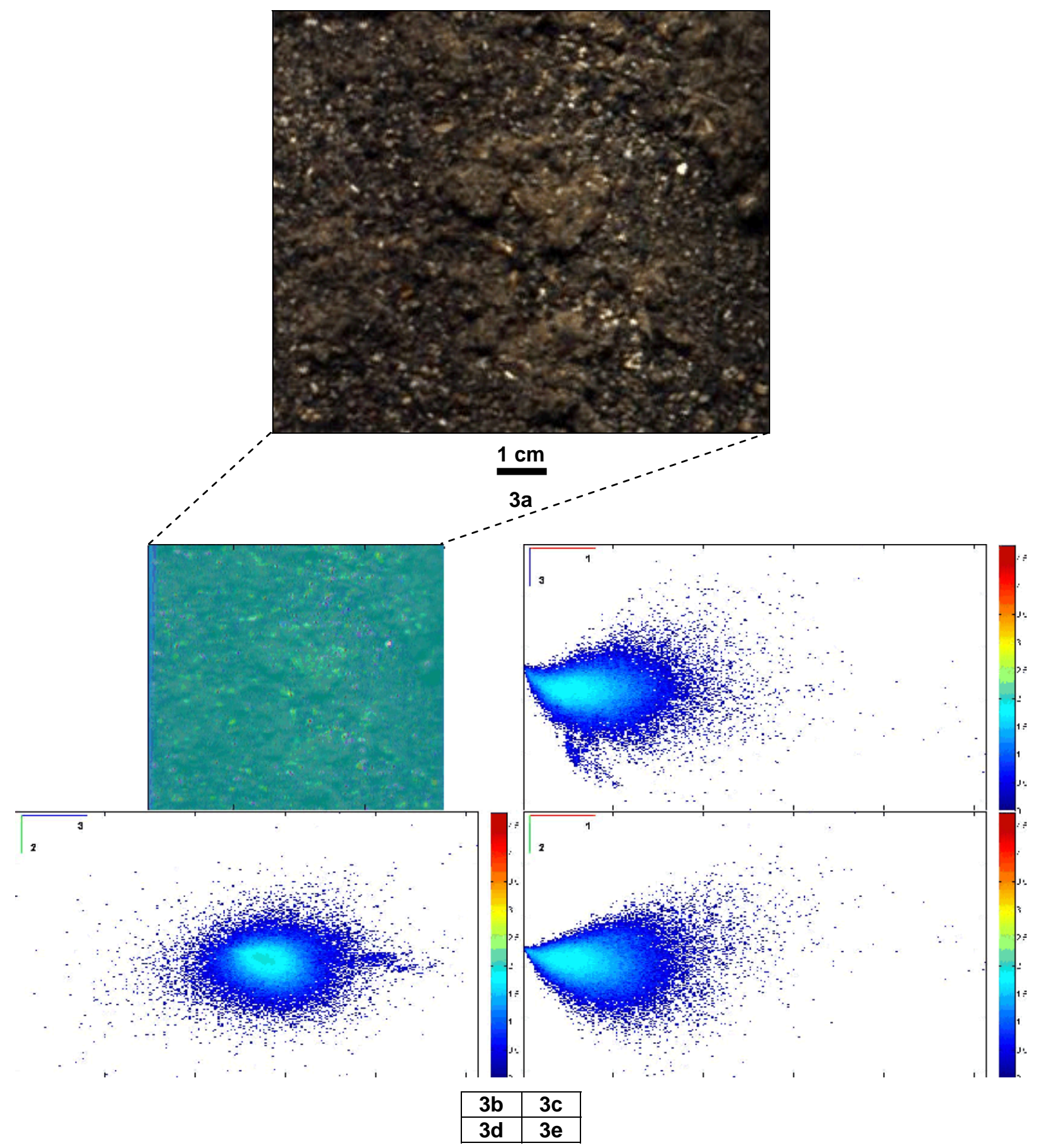

Fig. (3). Representation of the HSI data set, as acquired (3a), of bottom ashes product resulting from a wet processing resulting from the Amsterdam MSW incinerator. 3b: corresponding false colour image embedding the results of all the three score plots, that is 3c: [1-3], 3d: [3-2] and 3e: [1-2] related to PC1, PC2 and PC3 components as resulting from the application of the HPCIA. The presence of residual organic matter is clearly evidenced (presence of light areas on the false color image).

successful but costs and safety problems related to the utilization of this approach practically inhibited the further development. Therefore, the identification and the further removal of ceramic glass from the glass waste stream has long been, and also continue to represents, a challenge for glass recyclers.
Results: The comparison between glass and ceramic glass spectra showed as the two materials are characterized by a different spectral signatures. Such a difference is better evidenced increasing the investigated wavelengths range, that is moving from the $400-1000 \mathrm{~nm}$ range to $1000-2500$ $\mathrm{nm}$ range and over. Recognition is always performed 


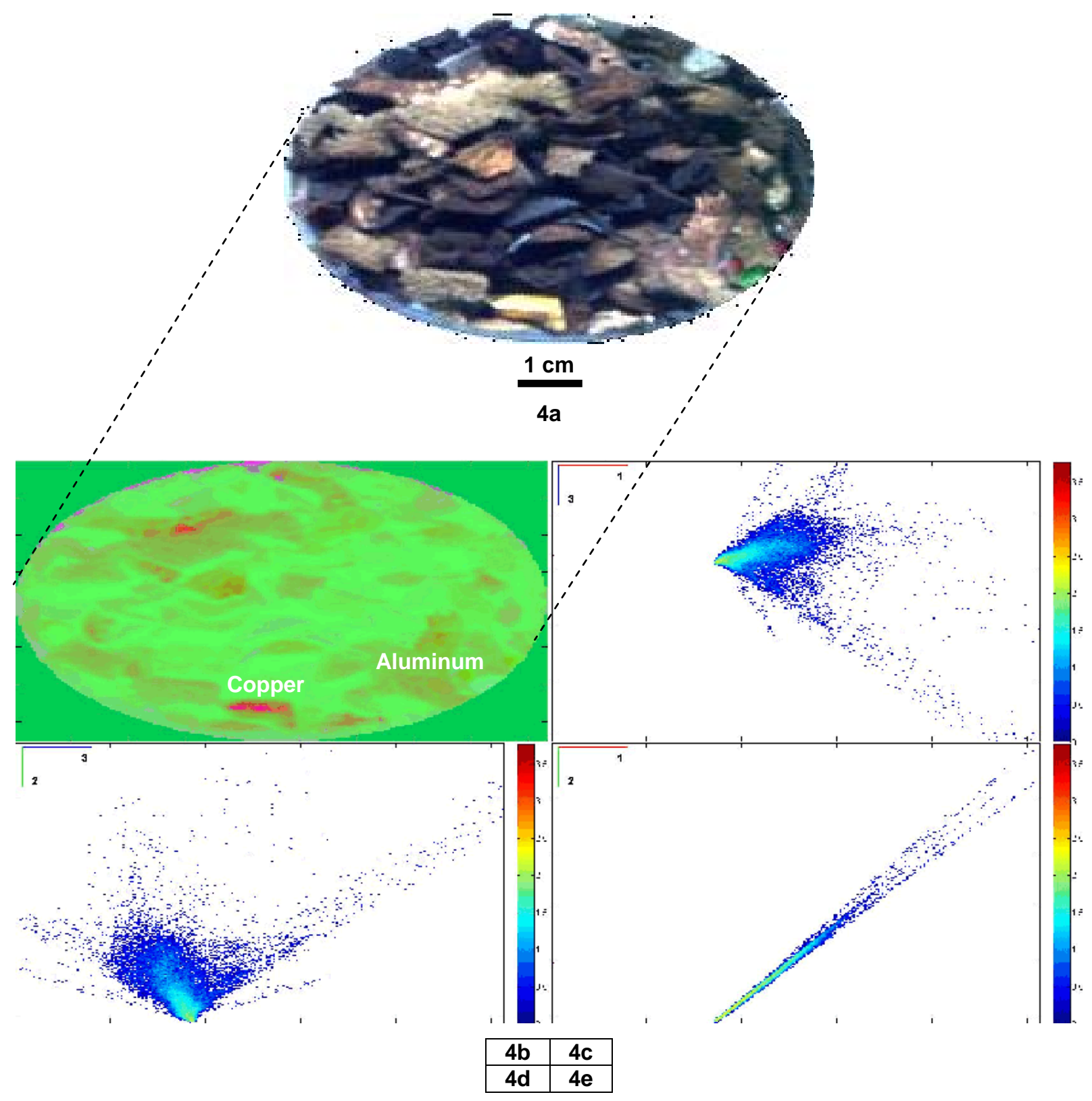

Fig. (4). Representation of the HSI data set, as acquired (4a), of fine, $-10 \mathrm{~mm}$, fluff product resulting from ASR processing. 4b: corresponding false colour image embedding the results of all the three score plots, that is $\mathbf{4 c}$ : [1-3], 4d: [3-2] and 4e: [1-2] related to PC1, PC2 and PC3 components as resulting from the application of the HPCIA. The presence of contaminants (Aluminum and Copper) is clearly evidenced by the procedure.

selecting specific wavelength ratios [29]. Also in this case the possibility to spatially identify the different fragment typologies (Fig. 5) opens new perspective in on-line automated sorting, not only to identify and separate ceramic glass fragment from glass, but also to perform a much more reliable and efficient separation of cullets by colors.

\section{IMPURITIES DETECTION IN SECONDARY PLASTICS}

One of the most important consequences of switching from post-industrial waste to complex waste, as the case of secondary plastics is that the process and quality control become essential technologies to keep track of changes of the input wastes, in terms of the amounts of valuable 


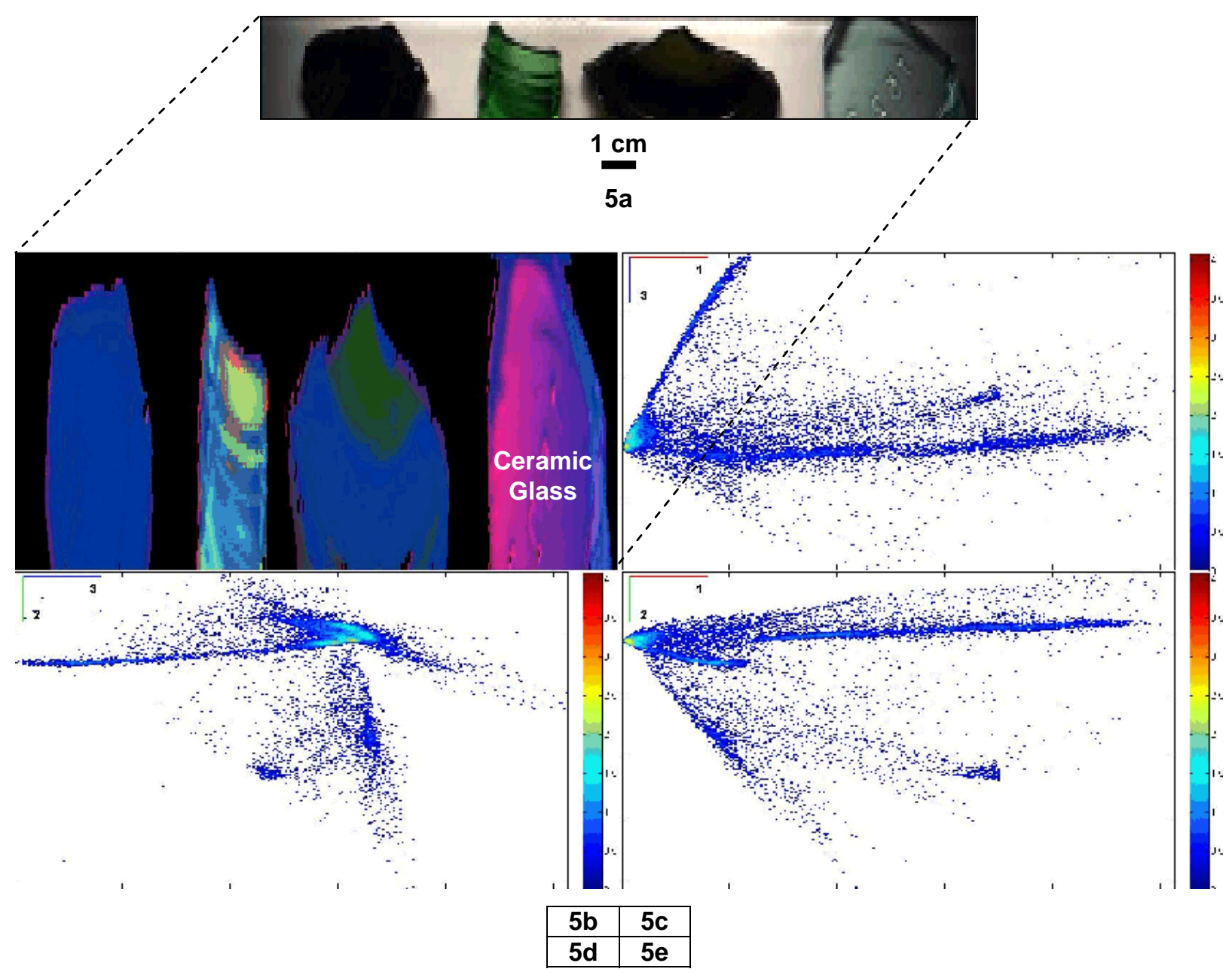

Fig. (5). Representation of the HSI data set, as acquired (5a), of different cullets resulting from a recycling process. The fragment on the right end side of the image is ceramic glass. 5b: corresponding false colour image embedding the results of all the three score plots, that is 5c: [1-3], 5d: [3-2] and 5e: [1-2] related to PC1, PC2 and PC3 components as resulting from the application of the HPCIA. Contaminant (ceramic glass) can be easily identified.

materials, major contaminants (rubber, wood, etc.) concentrations and particle size distribution of the input waste. Process control and quality control are equally vital to guarantee a consistent product quality, since the feed contains large amounts of contaminants and even small hickups of the process can deliver significant amounts of these contaminants to product bins and destroy the quality of the stored material. Furthermore the knowledge of feed streams composition, in terms of polluting materials, is a key issue to perform a correct regulation of the separation devices, whose performance are obviously influenced by input materials characteristics. This aspect is of particular importance for secondary plastic waste streams and when Magnetic Density Separation (MDS) architecture is utilised. Compositional variations of flows, in fact, can strongly affect MDS performance and the overall quality of the final products.

Therefore a precise and on-line assessment of composition of the process stream is of great importance for both the recycling and compounder industry in the transition to the recycling of post-consumer wastes. The former needs it to monitor the separation process. The latter demands it for the most accurate (and fast) composition assessment to calibrate the extruders (equipment) and to fulfil customers' requirements as well. Hence fast on-line assessment is a key issue to increase the value of secondary polyolefins.

The state-of-the-art analysis of PP or PE concentrates in terms of the concentration of the other polyolefin as well as non-polyolefin contaminants is currently obtained by means of hand-sorting and thermal analysis (Differential Scanning Calorimeter: DSC) of samples in laboratory. Other methods are Crystallization Analysis Fractionation (CRYSTAF), Infra-red Spectroscopy (IR) and Temperature Rising Elution Fractionation (ETREF). No one of these methods is suitable and accurate for the required on-line QICSL assessment and therefore new sensor technologies should be investigated and innovative control architectures and logics developed to quantify the concentration of contaminants in each of the products. 


\section{HYPERSPECTRAL MONITORING OF SECONDARY PLASTICS}

Hyperspectral cameras are able to deliver a wide spectrum of information from particulate solids streams. Wavelength intervals are usually those ranging between $400-$ $700 \mathrm{~nm}, 400-1000 \mathrm{~nm}$ and $1000-1700 \mathrm{~nm}$. Spectra are correlated to particles composition. Together with spectral response, other parameters are collected, as particles morphological and morphometrical attributes distribution, spatial and temporal fluctuations of the particles streams, etc. The development beyond the state-of-the-art will be to interpret the possibilities of HSI in determining the quality of feed and product stream in the recycling of secondary plastics and translate the images into the parameters that are requested by recycling operation, both in terms of control strategies set up and product quality assessment. Tests have been thus carried out to verify the practical utilization of hyperspectral cameras in respect of: i) secondary plastics feed characterization (particulate solids composition, that is different polymers and contaminants identification), ii) quality of the different flow streams resulting from specific processing actions (presence of contaminants and/or "pollutants") and iii) identification of PE and PP particles to set up new sorting strategies for their recovery.

\subsection{Laboratory Set Up, Spectral Device, Spectra Acquisition and Analysis}

The spectral analyses have been carried out utilizing the detection architecture reported in Fig. (6). The acquisition architecture allow to perform a progressive and continuous horizontal translation of the sample and the "synchronized" acquisition (at a pre-established step) of the spectra. The sensing device being constituted by an ImSpector $^{\mathrm{TM}}$ V10E (Specim, Finland) working in the visible-near infrared spectral range $(400-1000 \mathrm{~nm})$, with a spectral resolution of $2.8 \mathrm{~nm}$ and a spatial resolution less than $9 \mu \mathrm{m}$ [32].

The spectrograph is constituted by optics based on volume type holographic transmission grating (Fig. 7).

The grating is used in patented Prism-Grating-Prism construction (PGP element) characterized by high diffraction efficiency, good spectral linearity and it is nearly free of geometrical aberrations due to the on-axis operation principle. A collimated light beam is dispersed at the PGP so that the central wavelength passes symmetrically through the grating and prisms and the short and longer wavelengths are dispersed up and down compared to central wavelength. This results in a minimum deviation from the ideal on-axis condition and minimizes geometrical aberrations both in spatial and spectral axis. The availability of a detector constituted by a linear array of sensing elements, each one able to detect the spectral components of the corresponding investigated constituting domains of the object, could permit to measure the optical spectrum components and the spatial location of an object surface. The equipment and the related measuring procedures are based on such a principle. This target has been realized coupling together a dispersive stationary spectrograph and a CCD detector. The resulting information is thus constituted by a digital image where each column represents the discrete spectrum values of the

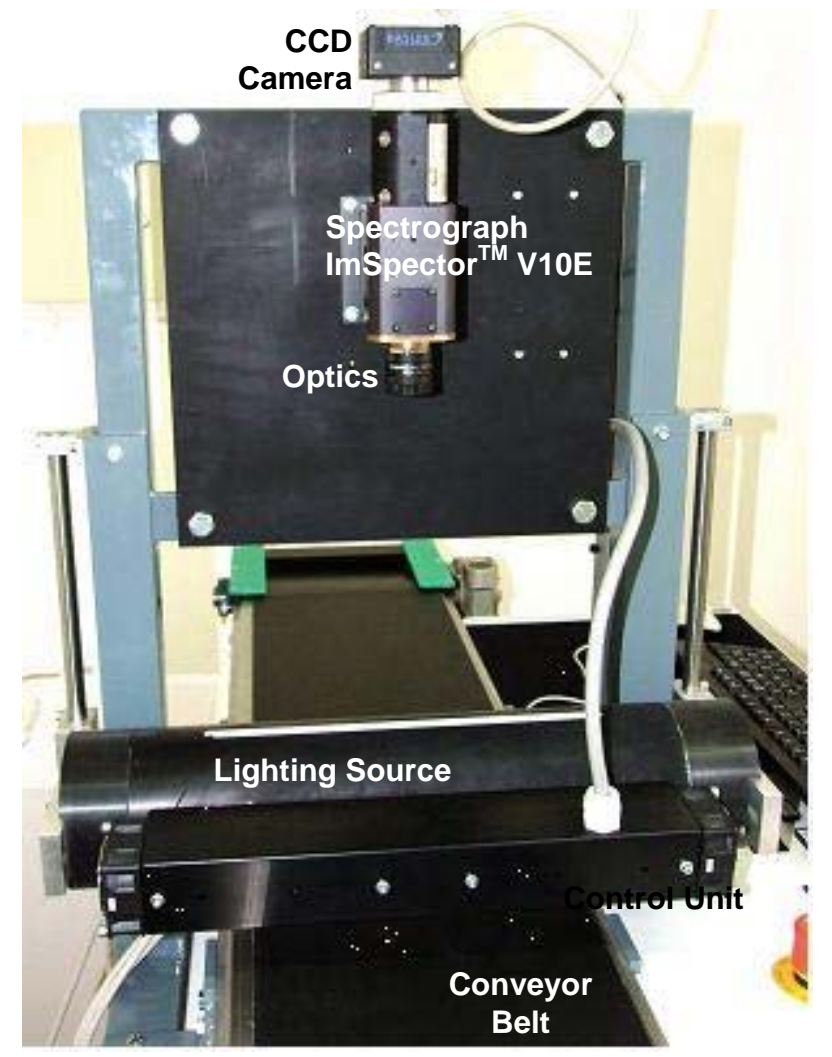

Fig. (6). Hyperspectral Imaging (HSI) based architecture utilized to acquire secondary plastic particles flow streams spectra.

corresponding element of the sensitive linear array. Such an architecture allows with a "simple" arrangement of the detection device ("scan line" perpendicular to the moving direction of the objects) to realize a full and continuous control. Differently from other detection systems, based on spectroscopy, the system offers several advantages: i) reduced measurement time, ii) no scanning movement and iii) simultaneous measurement over a line area. Line spectrometer is based on the detection, along a pre-defined alignment, of the spectra related to each element identified on the alignment itself. The length $\left(\mathrm{L}_{\mathrm{i}}\right)$ and the width $\left(\mathrm{W}_{\mathrm{i}}\right)$ of the "scene line" imaged at a time is thus determined by the slit length (Ls) and width $\left(\mathrm{W}_{\mathrm{S}}\right)$, by the lens focal length (f) and by the distance between investigated object and lens (D): $\mathrm{L}_{\mathrm{i}}=\mathrm{L}_{\mathrm{S}} \mathrm{D} / \mathrm{f}$ and $\mathrm{W}_{\mathrm{i}}=\mathrm{W}_{\mathrm{S}} \mathrm{D} / \mathrm{f}$. Spatial resolution along the image line is determined by the camera pixel size and point spread size of the optics. Illumination is a crucial part of the real time spectral imaging system. Usually experimental tests are needed to define the optimal lighting. Line lighting, as energizing source with uniform spatial distribution, is the most efficient solution. A preliminary step necessary to carry on, when an imaging spectrometry data handling and processing has to be applied is represented by calibration. Usually, it is performed in three steps, that is: i) spectral axis calibration with spectral lamps (i.e. $\mathrm{Hg}, \mathrm{Ne}, \mathrm{Ar}$ and $\mathrm{Kr}$ ); ii) dark image acquisition and storage and iii) measurements and storage of "white reference image". After the calibration phase: i) the image spectra is acquired and ii) the reflectance $\left(\mathrm{R}_{\mathrm{ci}}\right)$ (at wavelengths $\mathrm{i}$ and spatial pixels $\mathrm{c}$ of interest) is computed (Eq. 1): 


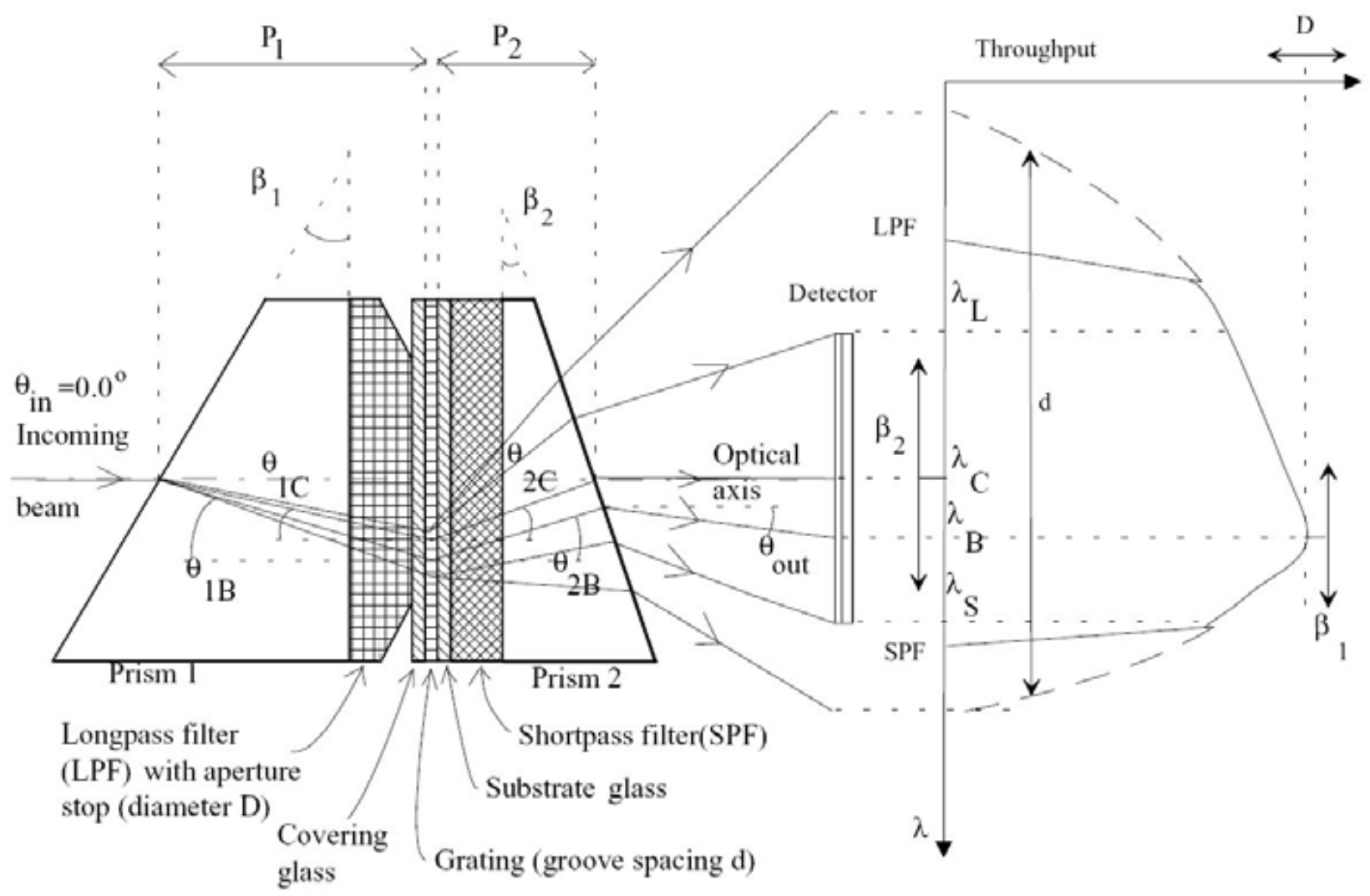

Fig. (7). Operating principle of a PGP spectrograph. $\lambda_{S}$ : shortest wavelength, $\lambda_{C}$ : central wavelength, $\lambda_{L}$ : longest wavelength, $P_{1}$ and $P_{2}$ : central thickness of the halves of the PGP, $\beta_{1}$ and $\beta_{2}$ : vertex angles of the prism, $\theta_{1 \mathrm{~B}}$ and $\theta_{2 \mathrm{~B}}$ : angles of incidence of the grating for the Bragg, $\lambda_{B}$, and central, $\lambda_{C}$, wavelengths in prism material, $\theta_{2 B}$ and $\theta_{2 C}$ : output angles from the grating in the prism material, $\theta_{\text {out }}=\theta\left(\lambda_{\mathrm{x}}\right)_{\text {out }}=$ output angle from PGP, d: grove spacing of the grating, D: diameter of the aperture stop.

$\mathrm{R}_{\mathrm{ci}}=\left[(\text { sample })_{c i}-(\text { dark })_{c i}\right] /\left[(\text { white })_{c i}-(\text { dark })_{c i}\right] \quad$ Eq. 1

Such a procedure enables to compensate the offset due to CCD dark current and separates the sample reflectance from the system response.

Analysis have been carried out performing a characterization of the "shape" of the entire detected spectra and/or identifying, at specific wavelengths, peaks or valley characterizing the detected signature and to verify, adopting a PCA the possible correlation existing among detected spectra, sample textural attributes, presence, characteristics and localization of the different materials and/or contaminants. A PCA is an orthogonal linear transformation of the data to a new coordinate system where the greatest variance by any projection of the data comes to lie on the first coordinate (first principal component, PC1), the second greatest variance on the second coordinate (PC2), and so on. PCA can be used for dimensionally reduction in a data set while retaining those characteristics of the data set that contribute most to its variance, by keeping lower-order principal components and ignoring higher-order ones [33, 34].

\subsection{Sample Preparation}

Secondary plastics resulting from shredding and sieving have been utilized to perform the tests (Fig. 8). After sieving

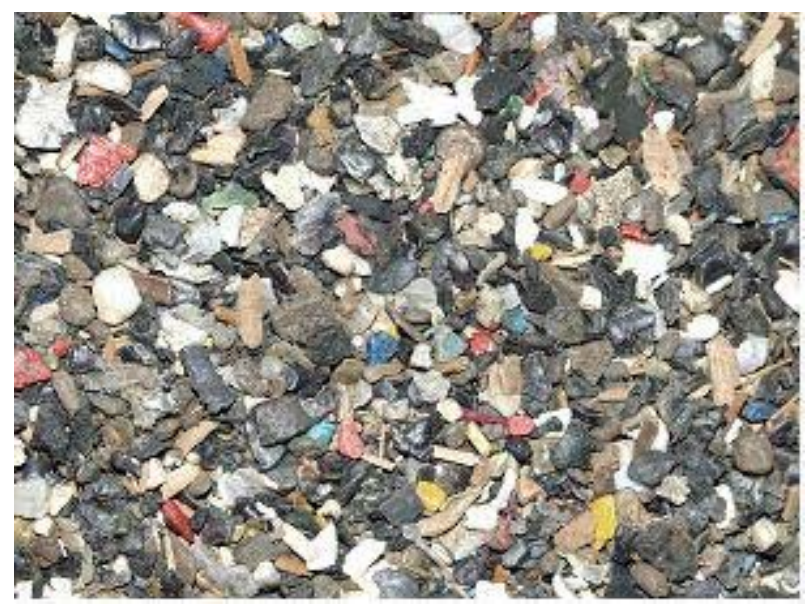

\section{$1 \mathrm{~cm}$}

Fig. (8). Different materials constituting the secondary plastics waste product after shredding and sieving.

at $2 \mathrm{~mm}$ the retained product was hand sorted in order to obtain reference classes of the different impurities as: rubber, wood, stone and metal. In every mixture, plastic constitutes the main material. Contaminants are present only in small amounts. The plastic fraction was then subjected to a sink- 


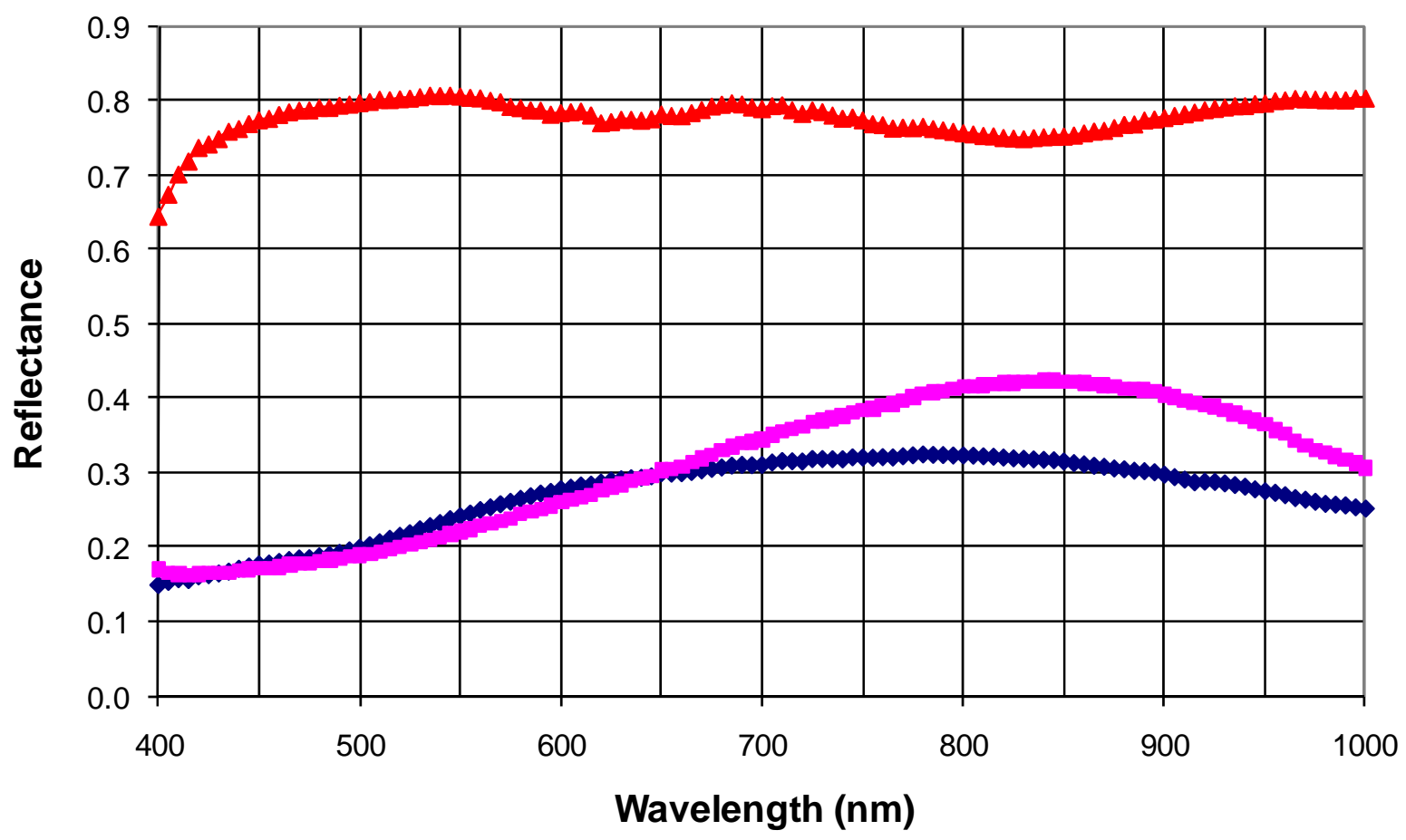

$\multimap$ Foam $\rightarrow$ Wood $\rightarrow$ Aluminium

Fig. (9). Average reflectance spectra in the VIS-NIR field (400-1000 nm) of the different contaminants detected, inside the investigated secondary plastics flow streams, adopting the HSI based architecture.

float separation at a cut density of $1000 \mathrm{~kg} / \mathrm{m}^{3}$ in water. This approach was followed to separate polyolefins (float fraction: $<1000 \mathrm{~kg} / \mathrm{m}^{3}$ ) from heavy plastics (sink fraction: $>1000 \mathrm{~kg} / \mathrm{m}^{3}$ ). Sample was then subjected to several sinkfloat separation stages (using the static bath method at various cut densities in water and water-ethanol mixtures, at room temperature) to obtain classes of products characterized by different density distribution. The application of this separation strategy produced as result different products, that should be representative of: heavy plastic (density $>1000 \mathrm{~kg} / \mathrm{m}^{3}$ ), HDPE (density between 930 and $1000 \mathrm{~kg} / \mathrm{m}^{3}$ ), LDPE (density between 915 and 930 $\mathrm{kg} / \mathrm{m}^{3}$ ) and PP (density $<915 \mathrm{~kg} / \mathrm{m}^{3}$ ). The hyperspectral approach was thus applied: to investigate the sensitivity of the method in respect of both the waste plastic feed and the different flow streams resulting from processing stream to characterize, identify the different organic based materials (plastics, foams, rubber, tires, etc.) and contaminants (wood, finer fractions and metals).

\subsection{Results}

The acquired reflectance spectra for the particulate solids constituting the feed allow identifying the different materials constituting the secondary plastics flow stream. Spectral plots clearly show as different materials present a different spectral signature (Figs. 9, 10). Unsorted plastics (PVC, PET, PE, PP, etc.), independently from their colors, are easily identifiable in respect of contaminants as wood, foam and aluminum. The adoption of an HPCIA, allows not only to identify pollutants but also to determine their topological assessment on the sample surface (Fig. 11). The analysis of the image related to PC1, PC2 and PC3 components permits, in fact, to detect presence, typology and position of different non plastic materials (contaminants) (Fig. 12). It is thus possible to quantitatively identify undesidered particles and, thanks to the possibility to know their relative position, to implement automatic sorting strategies for their removal, fulfilling this way the goal to define innovative QICSL.

\section{THE ROLE OF THE HYPERSPECTRAL APPROACH INSIDE THE RECYCLING ROUTE}

The results demonstrated as the proposed innovative control approach perfectly fits with the concept outlined by the CIM, that is [3]:

"The cyclic innovation model can be applied to facilitate the implementation and adaption of new technologies with the potential to turn them into a profitable green business. A strategy has to be designed to move along the transition path from the current situation (today) to the image of the future (when the new technology is used) with minimum delay. There will be a number of obstacles along this transition path that must be overcome to realize a fast implementation. These obstacles 


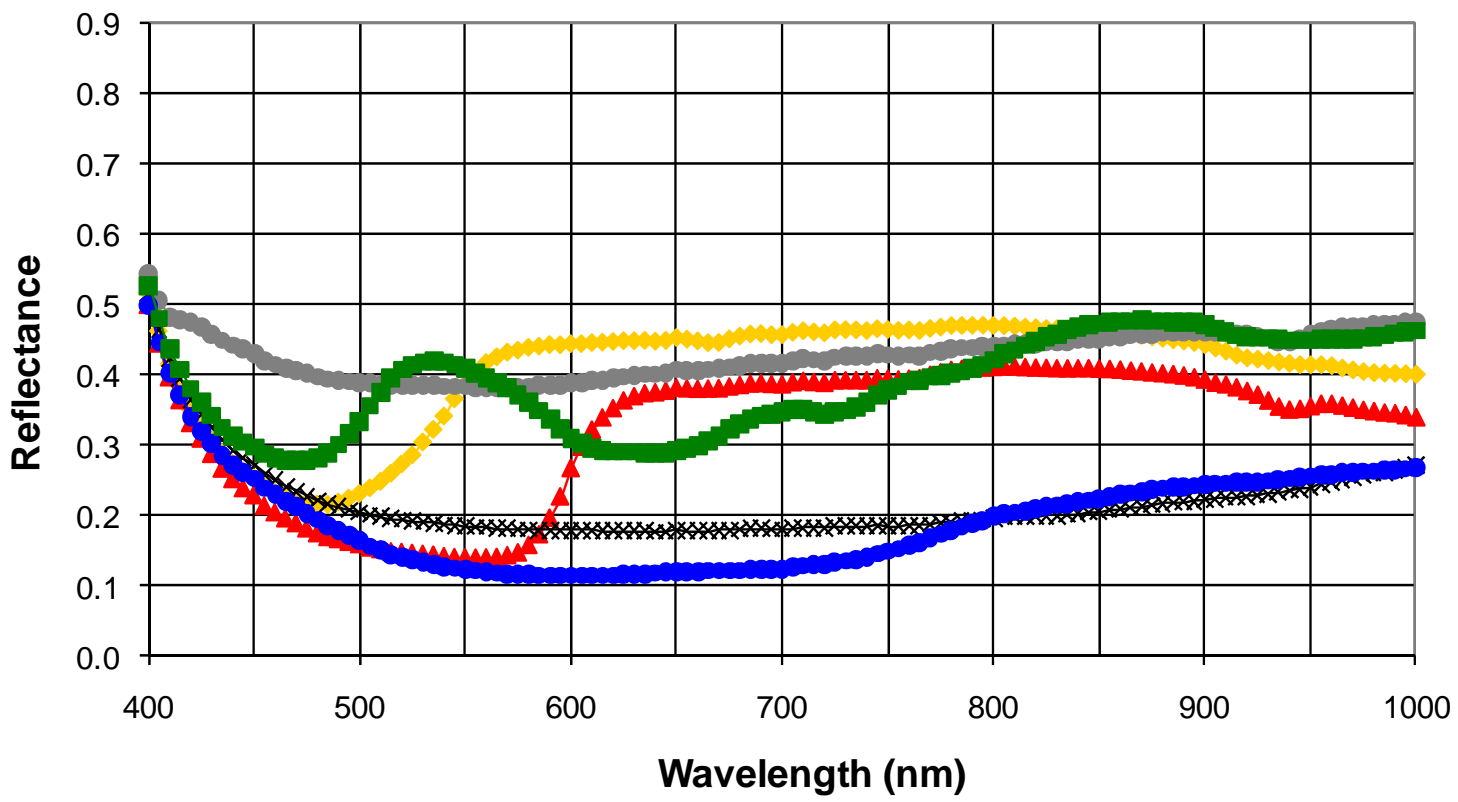

$\rightarrow-P$ yellow $\rightarrow$ P_red $\rightarrow$ P_black $\rightarrow-P \_$white $\rightarrow$ P_blue $\rightarrow$ P_green

Fig. (10). Average reflectance spectra in the VIS-NIR field (400-1000 nm) of different plastic materials of different colour, identified inside the investigated secondary plastics flow streams, adopting the HSI based architecture. Plastics spectral signatures are quite different from those representative of the contaminants (foam, wood and Aluminum) (Fig. 8).

must be identified and translated into challenges that need to be addressed".

With reference to HSI based quality-control strategies the main obstacles is to switch the applications from high-tech industrial sectors to a sector, the solid waste recycling, that is objectively not so advanced in terms of technologies utilized and applied research performed. Furthermore, the lack of knowledge and the fear to introduce new processing logics inside consolidated ones, where the role of "humans" continue to be, in some cases, preeminent, constitutes a serious "psychological" obstacle to the introduction of this new technology. An aid in implementing the HSI approach can be represented by the intrinsic characteristics of the technology both in terms of modalities of information acquisition and detection architecture. The approach, in fact, can be easily implemented, in technological terms, practically in every plant where a waste flow stream is accessible in terms of inspection possibilities. Such a situation is practically always realized inside every solid waste recycling plant. In a CIM perspective [3] this means that the adoption of HSI based logic in product control affects mainly two classes of innovation, that is: i) the Integrated Engineering Cycle (Technological Renewal $\leftrightarrow$ Product Development) and ii) the Differentiated Services Cycle (Product Development $\leftrightarrow$ Market Transitions). Main and potential obstacles that could affect the two classes of innovation are:

- $\quad$ the correct finalization of the scientific and technical efforts to develop the new quality control device, that is the development of a system able to recognize plastics materials and/or polluting ones, in order to be utilized not only as a sorting engine, but also as the analytical core to perform waste products quality certification for the different stage of processing,

- $\quad$ the possibility to assure a robust production both in terms of quality and quantity of the recycled product. Such a goal should also take into consideration the possibility to define new classes of recycled products, not only in composition, but also in terms of their physical attributes (size, shape, morphology) according to the innovative recycling process embedding the HSI approach.

All these aspects are considered and faced inside the European FP7 Project: W2Plastics [35].

\section{CONCLUSIONS}

HyperSpectral Imaging (HSI) approach was discussed with reference to the possibility to perform the recognition of impurities inside secondary plastics flow streams in order to certify and to classify waste plastics fed to recycling plants and to perform a full control of the resulting processed fractions. Starting from the experience carried out in previous studies, always addressed to waste materials to recycle, as bottom ashes, fluff and cullets, a full HSI approach was developed to verify the reliability of the method: 


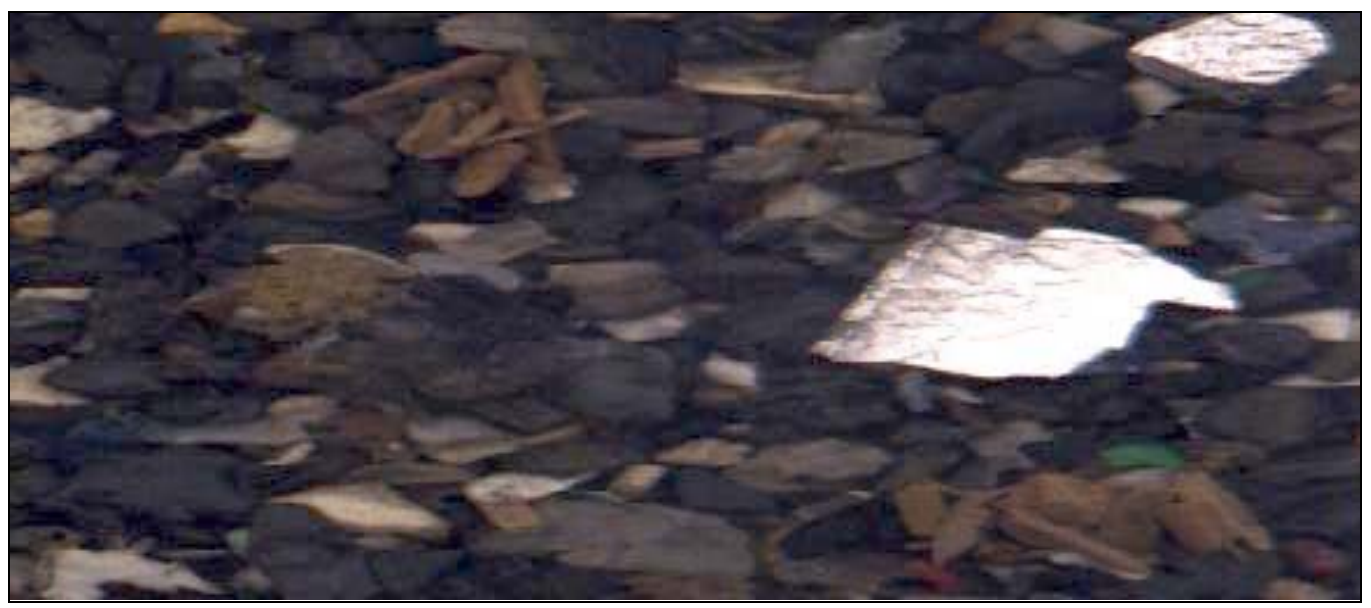

$1 \mathrm{~cm}$

$11 a$

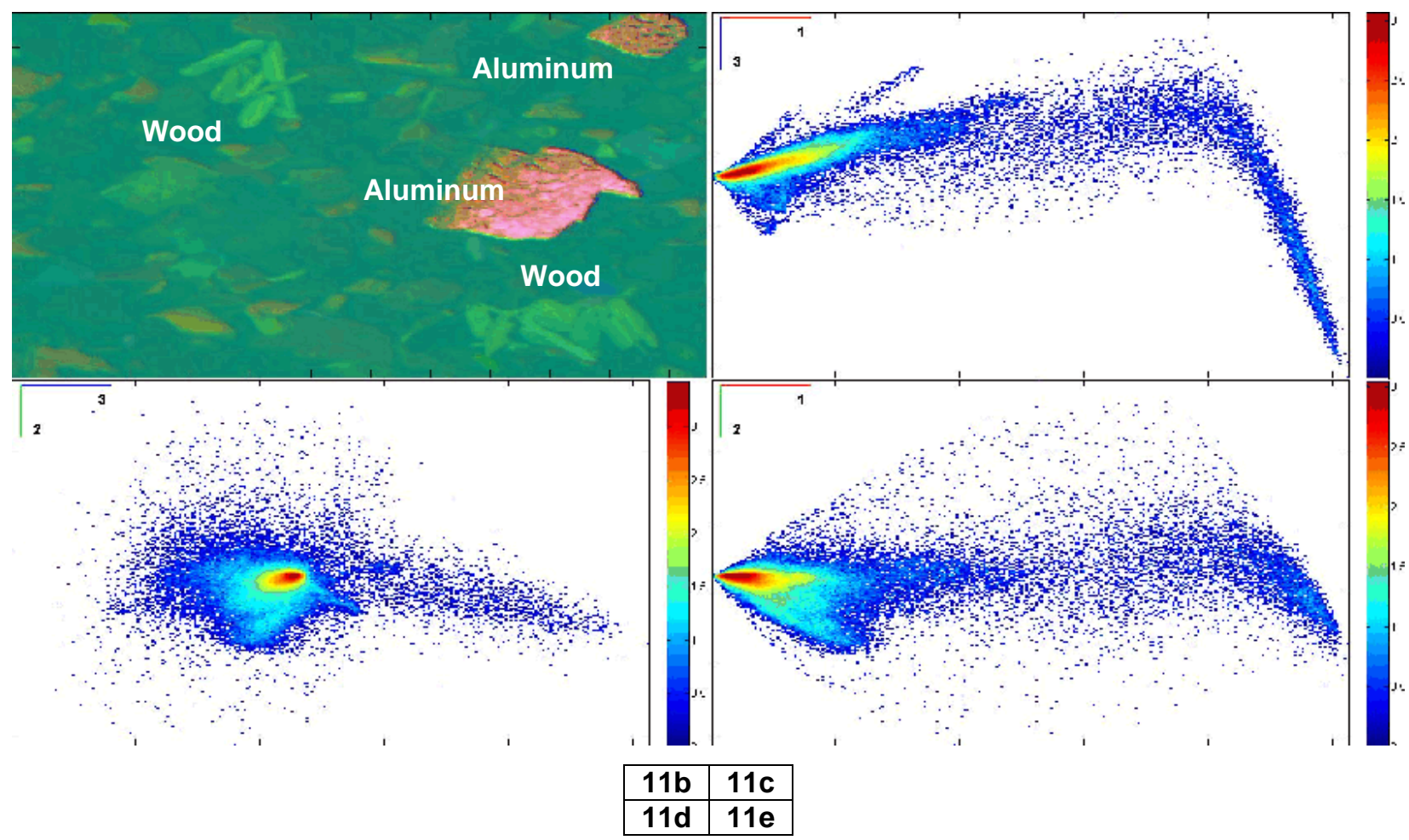

Fig. (11). Representation of the HSI data set, as acquired (11a), of a sample of secondary plastics fed to the MDS processing. 11b: corresponding false colour image embedding the results of all the three score plots, that is 11c: [1-3], 11d: [3-2] and 11e: [1-2] related to PC1, PC2 and PC3 components as resulting from the application of the HPCIA. Contaminant (ceramic glass) can be easily identified.

- in order to identify specific spectral attributes of the different materials constituting the plastic waste streams, in order to perform the recognition of the different constituents (plastics and impurities) and

- to establish, through the application of a Principal Component Analysis (PCA), possible correlation among detected spectra, sample textural attributes, presence, characteristics and localization of pollutants.

Results showed as HSI can represent an optimal, reliable and low costs answer to reach the previous mentioned goals and represents, for the waste sector, a big-step-forward inside quality-inspection-control-strategies/logics (QICSL). Finally the study was evaluated inside the Cyclic Innovation Model (CIM), a new conceptual framework developed at 


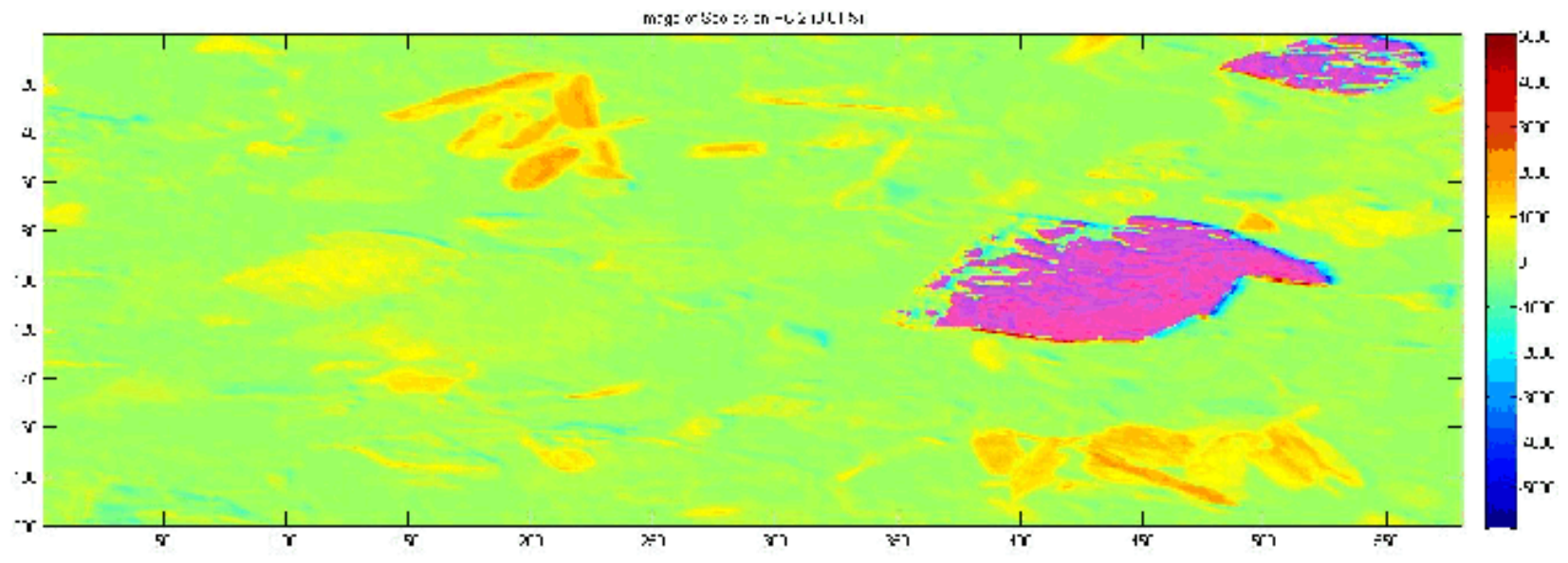

$12 a$

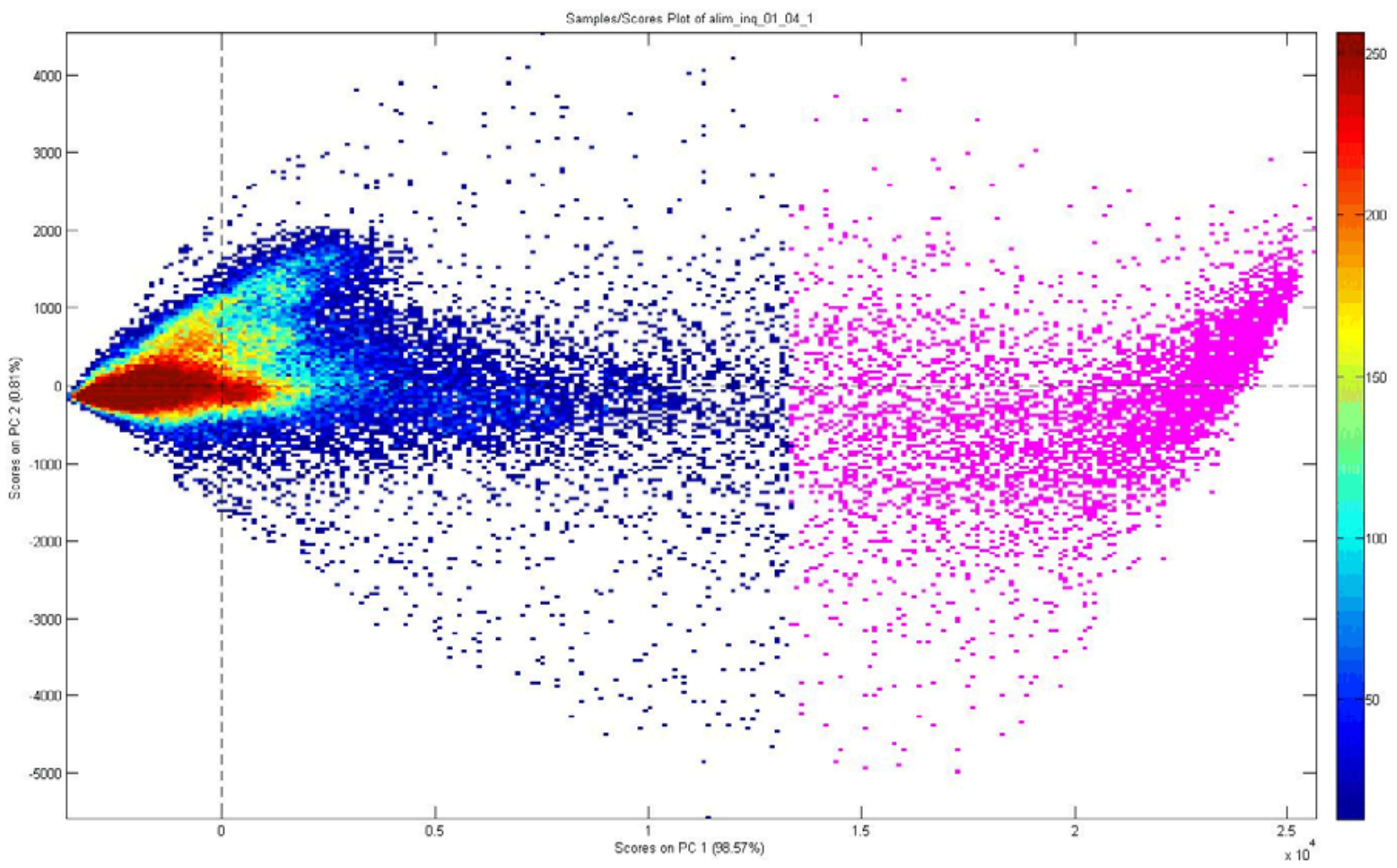

$12 b$

Fig. (12). Image on scores on PC2 (12a) of the HSI data set of Fig. (11). Samples/Scores Plot PC1-PC2 clearly outlines the correspondence existing among typology of contaminants and their "mapping" inside the plot (12b).

Delft University of Technology. Such evaluation demonstrated as the proposed approach, for its characteristics, can represent an example of innovative technology that can be applied to implement new separation technologies, as Magnetic Density Separation (MDS), to turn them into a profitable green business.

\section{ACKNOWLEDGEMENTS}

The study was developed with thanks to the financial support of the European Commission in the framework of the FP7 Collaborative project "Magnetic Sorting and Ultrasound Sensor Technologies for Production of High Purity Secondary Polyolefins from Waste (W2Plastics)", Grant Agreement No. 212782. 


\section{REFERENCES}

[1] J. E. Johansson, "Plastics - the compelling facts and figures", in the $6^{\text {th }}$ IdentiPlast Biennial Conference on the Recycling and Recovery of Plastics, 2007.

[2] G. Bonifazi, N. Picone, S. Serranti, E. J. Bakker and P. Rem, "High purity polyolefins recycling by melt separation", Metalurgia Int., vol. 14, pp.17-20, 2009.

[3] E. J. Bakker, A. J. Berkhout, L. Hartmann and P. C. Rem, “Turning magnetic density separation into green business using the cyclic innovation model”, Open Waste Manage. J. Special Issue, 2010 , vol. 3, pp.36-46.

[4] www.delphi.tudelft.nl

[5] H. W. Chesbrough, "Open Innovation: The New Imperative for Creating and Profiting from Technology", Harvard Business School Press, Watertown, MA, USA, 2003

[6] E. V. Hippel, "Democratizing Innovation", MIT Press, Boston, MA, USA, 2005.

[7] A. J. Berkhout, P. V. D. Duin, D. Hartmann and R. Ortt, "The Cyclic Nature of Innovation: Connecting Hard Sciences with Soft Values", Advances in the Study of Entrepreneurship, Innovation and Economic Growth, Elsevier, Amsterdam, vol. 17, 2007.

[8] H.F. Grahn and P. Geladi, "Techniques and Applications of Hyperspectral Image Analysis", John Wiley and Sons LTD, England, 2007.

[9] A. F. H. Goetz, G. Vane, T. E. Solomon, B. N. Rock, "Imaging spectrometry for earth remote sensing", Science, vol. 228, pp. 1147-1153, June 1985.

[10] E. Hege, D. O'Connell, W. Johnson, S. Basty, and E. Dereniak, "Hyperspectral imaging for astronomy and space surveillance", Proc. SPIE, vol. 5159, pp. 380-391, January 2003.

[11] K. S. Wood, A. M. Gulian, G. G. Fritz and D. Van Vechten, "A QVD detector for focal plane hyperspectral imaging”. Astronomy. Bull. Am. Astronom. Soc., vol. 34, pp. 1241, 2002.

[12] S. Monteiro, Y. Minekawa, Y. Kosugi, T. Akazawa K. and Oda, "Prediction of sweetness and amino acid content in soybean crops from hyperspectral imagery", ISPRS J. Photogramm. Remote Sensing, vol. 62, no. 1, pp. 2-12, May 2007.

[13] V. Smail, A. Fritz and D. Wetzel, "Chemical imaging of intact seeds with NIR focal plane array assists plant breeding", Vib. Spectrosc., vol. 42, no. 2, pp. 215-221, November 2006.

[14] Y. Uno, S. Prasher, R. Lacroix, P. Goel, Y. Karimi and A. Viau, "Artificial neural networks to predict corn yield from Compact Airborne Spectrographic Imager data". Comput. Electron. Agric., vol. 47, no. 2, pp. 149161, May 2005.

[15] R. C. Lyon, D. S. Lester, E. N. Lewis, E. Lee, L. X. Yu and E. H. Jefferson, "Near-infrared spectral imaging for quality assurance of pharmaceutical products: analysis of tablets to assess powder blend homogeneity", AAPS PharmSciTech., vol. 3, no. 3, pp. 17, April 2002.

[16] O. Rodionova, L. Houmøller, A. Pomerantsev, P. Geladi, J. Burger, V. Dorofeyev, "NIR spectrometry for counterfeit drug detection: a feasibility study", Anal. Chim. Acta, vol. 549, no. 1-2, pp. 151-158, September 2005.

[17] Y. Roggo, A. Edmond, P. Chalus and M. Ulmschneider, "Infrared hyperspectral imaging for qualitative analysis of pharmaceutical solid forms", Analytica Chimica Acta, vol. 535(1-2), pp. 79-87, April 2005.

[18] D. Ferris, R. Lawhead, E. Dickman, N. Holtzapple, J. Miller and S. Grogan, "Multimodal hyperspectral imaging for the non invasive diagnosis of cervical neoplasia”, J. Lower Genit. Tract Dis., vol. 5, no. 2, pp. 65-72, December 2001.

[19] D. Kellicut, J. Weiswasser, S. Arora, J. Freeman, R. Lew, and C. Shuman, "Emerging technology: hyperspectral imaging", Perspect. Vasc. Surg. Endovasc. Ther., vol. 16, no. 1, pp. 53-57, January 2004.

[20] G. Zheng, Y. Chen, X. Intes, B. Chance and J. D. Glickson, "Contrast-enhanced near-infrared (NIR) optical imaging for subsurface cancer detection", J. Porphyr. Phthalocyanines, vol. 8, no. 9, pp. 1106-1117, December 2004

[21] A. A. Gowen, C. P. O’Donnel, P. J. Cullen, G. Downey, and J.M. Frias, "Hyperspectral imaging - an merging process analytical tool for food quality and safety control", Trends in Food Science \& Technology, vol. 18, pp. 590-598, December 2007.

[22] Lu, R. F. and Chen, Y. R., "Hyperspectral imaging for safety inspection of food and agricultural products", SPIE Conference on Pathogen Detection and Remediation for Safe Eating, Boston, November, 1998.

[23] http://www.silvereng.com/PDF/NEMO.pdf

[24] E. Lewis, J. Schoppelrei, E. Lee, and L. Kidder, "Near-infrared chemical imaging as a process analytical tool”, K. Bakeev (Ed.), Process analytical technology (pp. 187). Oxford: Blackwell Publishing, 2005.

[25] D. Pappas, B. W. Smith, and J. D. Winefordner, "Raman imaging for two-dimensional chemical analysis", Appl. Spectrosc. Rev., vol. 35, pp. 1-23, May 2000.

[26] K. Zuzak, M. Schaeberle, M. Gladwin, R. Cannon and I. Levin, "Non invasive determination of spatially resolved and timeresolved tissue perfusion in humans during nitric oxide inhibition and inhalation by use of a visible reflectance hyperspectral imaging technique. Circulation, vol. 104, 2905, January 2001.

[27] K. C. Lawrence, B. Park, W. R. Windham and C. Mao, "Calibration of a pushbroom hyperspectral imaging system for agricultural inspection", Trans. ASAE, vol. 46, no. 2, pp. 513-521, January 2003.

[28] I. T. Jolliffe, Principal Component Analysis, Springer-Verlag, New York, 2002.

[29] S. Serranti and G. Bonifazi, "Solid waste materials characterization and recognition by hyperspectral imaging based logics", The $2^{\text {nd }}$ Int. Symposium MBT 2007: Mechanical Biological Treatment and Automatic Sorting of Municipal Solid Waste. pp. 326-336, 2007.

[30] P. C. Rem, C.De Vries, L. A. van Koov, P. Bevilacqua and M. A. Reuter, "The Amsterdam pilot on bottom ash", Miner. Eng., vol. 17, pp. 363-365, February 2004

[31] T.P. R. de Jong and W.L. Dalmijn, "X-ray transmission imaging for process optimisation of solid resources", Paper no. 173 , Proceedings R'02 Congress, February 12-15, Recovery Recycling Re-integration, Geneva. EMPA, Peak Ltd., Switzerland, 2002.

[32] SSOM, Spectral Scanner Operative Manual (Version 2.0). DV Optics S.r.l., Italy. Available at: http://www.dvoptic.com/index. html, 2008.

[33] P. Geladi, "Chemiometrics is spectroscopy. Part 1. Classical chemometrics”, Spectrochim. Acta Part B, vol. 58. pp. 767-782, May 2003.

[34] P. Geladi, "Chemiometrics is spectroscopy. Part 2. Examples", Spectrochim. Acta Part B, vol. 59. pp. 1347-1357, September 2004. P.C. Rem, S. Venga and F. Di Maio, "High-Purity Products from Plastic Waste: the W2Plastics Project", International Conference on Materials Science \& Engineering (BraMat), Brasov, pp. 26-28, February 2009 